\title{
Identifcation of FHIR Usage Patterns in IHE Profiles
}

\author{
Martin STAEMMLER ${ }^{\mathrm{a}, 1}$ \\ ${ }^{a}$ University of Applied Sciences, Stralsund, Germany
}

\begin{abstract}
IHE profiles have been enhanced by FHIR based functionality, however relying on different approaches. Based on an analysis of the 33 IHE profiles using FHIR five patterns have been identified reflecting the approaches, namely (i) wrapping, (ii) adding of FHIR based actors and transactions, (iii) resource operation extension, (iv) purely FHIR based profiles and (v) content profiles relying on FHIR resources. In addition, both the maturity and the development of these profiles over time have been assessed.
\end{abstract}

Keywords. IHE profiles, FHIR resources, maturity, integration

\section{Introduction}

The use of FHIR resources within IHE profiles has increased over the last years and was well received in applications for health information exchange and providing structured data [1-3]. While several profiles have been extended by dedicated actors and transactions supporting FHIR only few have been created being purely FHIR based. As such, the approach on how FHIR is being used within IHE profiles varies.

The objective of this paper to identify different usage patterns and apply these in an analysis of FHIR based IHE profiles. In addition the status of IHE profiles using FHIR is analyzed with regard to the maturity level, release status, IHE domains and implementation.

\section{Methods}

The lists of IHE profiles [4, 5] identify 33 FHIR based profiles. They were reviewed to derive patterns regarding the approach for introducing FHIR into existing IHE profile or newly established IHE profiles. The following criteria were used: (i) providing FHIR functionality by means of wrapping IHE actors with FHIR based actors, (ii) adding new FHIR based actors and /or transactions to an existing IHE profile covering the full or only a part of the original profile's functionality, (iii) amendments for specific resource operations related e.g. to queries, (iv) FHIR based content profiles, (v) profiles supporting transitions from IHE XDS based data object content to FHIR based granular information representation, (vi) IHE domain assignment of the FHIR based profile, (vii) release status of the FHIR resources, (viii) IHE profile state (trial implementation

\footnotetext{
${ }^{1}$ Corresponding Author, Martin Staemmler, University of Applied Sciences, Stralsund, Germany, martin.staemmler@hochschule-stralsund.de.
} 
or final text) and (ix) its test record in connectathons, using the IHE Connectathon Result Browser. In addition, this review has been conducted in January 2020 and January 2021 allowing assessing the development over time for some of the criteria.

\section{Results}

Overall, five patterns for the usage of FHIR in IHE profiles have been derived.

\subsection{Pattern 1: Wrapping IHE actors to provide FHIR interfaces}

Figure 1 demonstrates the grouping of a IHE profile actor with a FHIR based actor to provide FHIR compliant interfaces at the source and consumer site.

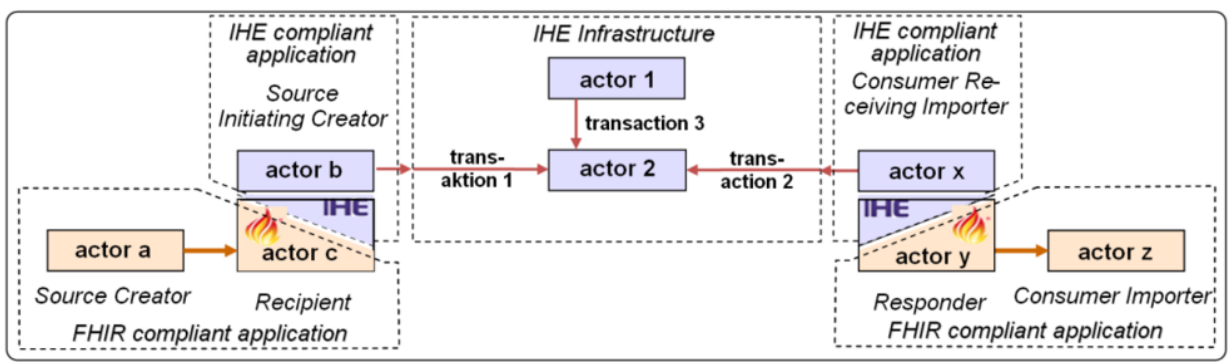

Figure 1. Grouping IHE profile actors with FHIR based actors.

The most typical example is the profile MHD (Mobile access to Health Documents) which was one of the first ones which made IHE XDS actors FHIR compliant.

\subsection{Pattern 2: Adding FHIR based actors or transactions to an existing IHE profile}

A more integrative pattern has been established with additional FHIR based actors allowing the full or partial functionality to be provided in the FHIR domain as well. For example, PDQm (Patients Demographics Query for Mobile) [7] amends the existing actors of PDQ (Fig. 2 left) with the transaction "Mobile Patient Demographics Query" and makes the two actors partially FHIR based (Fig. 2 right).

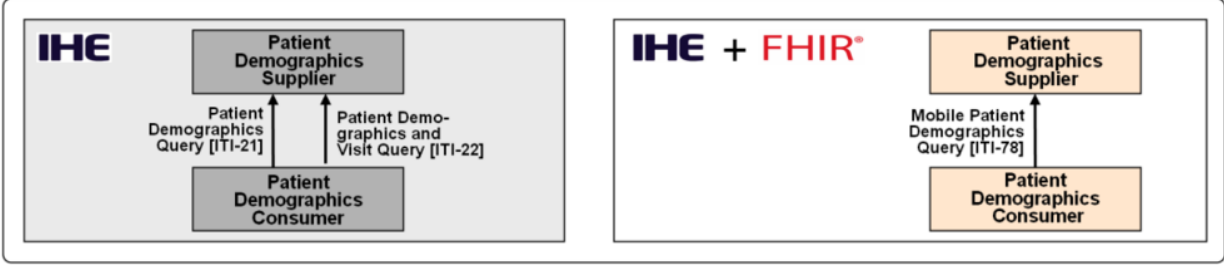

Figure 2. Adding FHIR based actors or transactions to an IHE profile

\subsection{Pattern 3: Specific amendments to FHIR resource operation}

Typical for FHIR resources the command $<$ base $><$ resource $>$ \$ $<$ operation $>$ allows the use of defined operations (e.g. \$validate) to be executed [8]. By adding 
a profile specific operation the functionality of a FHIR based resource is extended. For example, the IHE profile PIXm (Patient Identifier Cross-reference for Mobile) [4] allows to specify the scope of the cross-referencing in the FHIR Patient resource with the specific Patient\$ihe-pix operation.

\subsection{Pattern 4: New profiles using only FHIR based actors}

This pattern is characterized by a purely FHIR based approach, both with new actors and transactions and no obligation of grouping. For example, the profile QEDm (Query Existing Data for Mobile) with its actors "Clinical Data Consumer/Source" provides the transaction "Mobile Query Existing Data" [7]. Its function is comparable to the typical "XDS Registry Stored Query", however being fully FHIR based.

\subsection{Pattern 5: Content profiles using FHIR resources}

While IHE content profiles initially use representations like the Clinical Document Architecture (CDA) a comparable approach is possible with a set of bundled FHIR resources and the resource "Composition" to reflect the context of the information handled. A recent example is the content profile IPS (International Patient Summary) [7] which may include both, a FHIR and CDA representation.

\subsection{Pattern usage statistics}

Figure 3 shows the assignment of IHE profiles to the identified patterns. The analysis shows, that FHIR functionality is mostly embedded in the IHE profiles by adding actors and transactions, however in most cases following the Pareto principle of FHIR by aiming for only a partial functionality when compared to the IHE profile. Since some of the profiles are not yet fully specified a "not assigned" pattern had to be added.

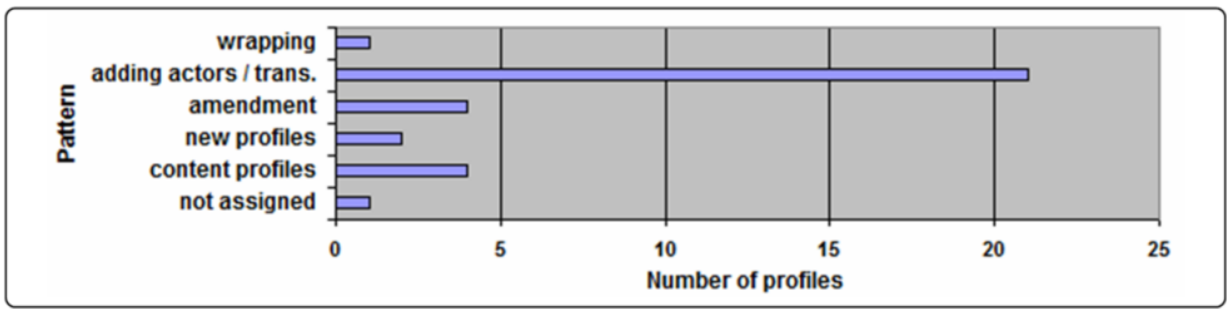

Figure 3. Number of profiles per pattern after assignment

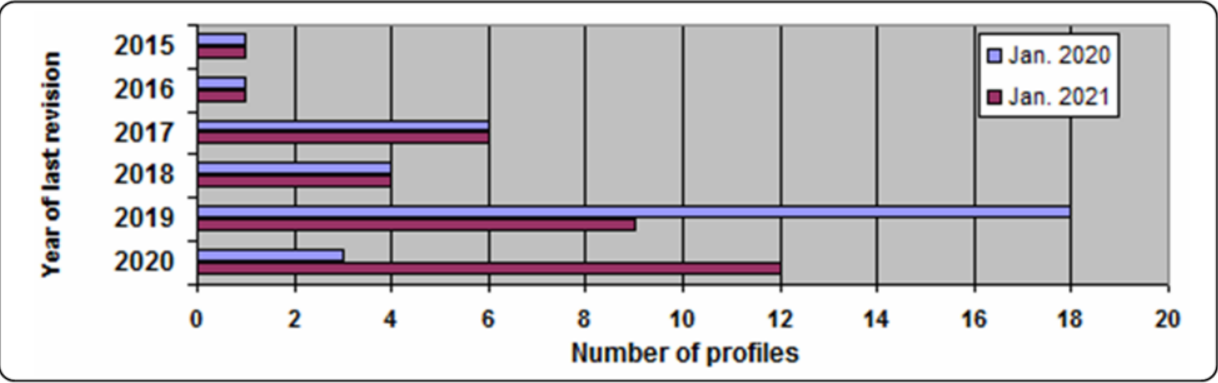

Figure 4. Development over time of IHE profiles using FHIR 


\subsection{Development and maturity of IHE profiles using FHIR}

Figure 4 confirms continuous development based on the design and revision dates mainly reflecting the year 2019 and 2020 as well as the ongoing advancement obvious from the two different time points.

Figure 5 represents the maturity of the FHIR resources. It has advanced to be more R4 based in 2021 than in 2020. The category of "unknown" is due to profile descriptions, which were not detailed enough to assign a specific release state.

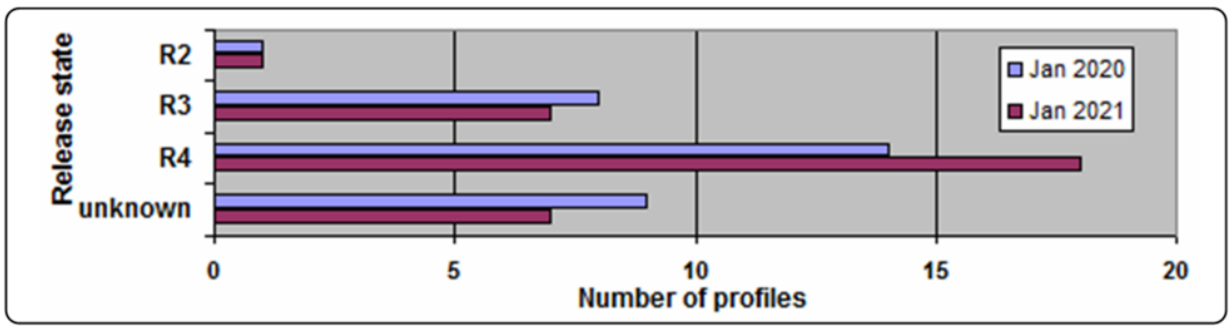

Figure 5. Maturity of IHE profiles using FHIR

\section{Discussion}

The five patterns are unevenly populated. For "wrapping" as a rather indirect approach its low scoring is reasonable. Amending the "operations available" for a FHIR resource poses the risk of implementation in FHIR servers and acceptance at a larger scale, despite being versatile. As such it has a limited use. Regarding "new profiles" and "content profiles" IHE is assumed to be at the verge of developing more profiles following these patterns. "Addition of actors / transactions" is the most widely pattern used. It could be further assessed with regard to the level of functionality provided in the FHIR range versus the IHE range. However, this would be difficult without available usage statistics from actual implementations.

A further criterion to discuss the implementation of IHE profiles is the number of vendors which tested a profile at Connectathons. Fig. 6 shows rather low numbers, with only few profiles tested by more than 10 vendors and the majority ( 22 of 33 profiles) not yet tested at all.

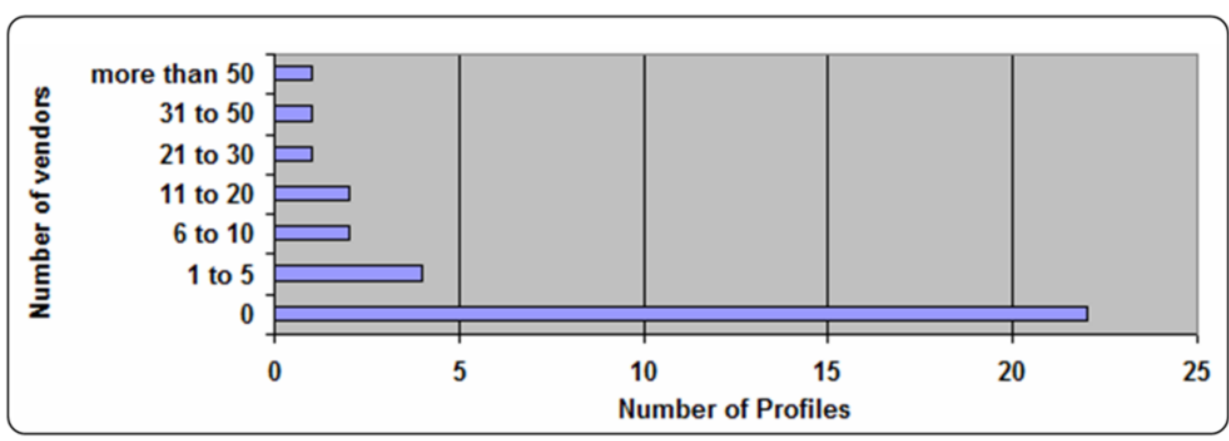

Figure 6. Number of vendors having tested FHIR based profiles up to January 2021 
Regarding the coverage of the IHE domains the ITI (IT-Infrastructure) with 35\%, PCC (Patient Care Coordination) with 30\% and QRPH (Quality, Research and Public Health) with $21 \%$ are the most active domains. The remaining percentages originate from the Pharmacy, Patient Care Devices and Radiology domains.

\section{Conclusions}

The five identified FHIR usage patterns in IHE profiles allow for monitoring the advancement of IHE profiles using FHIR. It is expected that the dominating pattern reflecting the addition of actors and transitions will stay in that role. However, the usage of the other patterns will increase based on more self standing profiles using FHIR. An indication for moving towards that objective is the profile MHDS (Mobile Health Document Sharing) [9] which outlines a whole FHIR based ecosystem for secure and authenticated health information exchange purely based on FHIR enabled IHE profiles. Its deployment allows both for desktop as well for mobile applications. The need for structured data to meet the medical, reporting and research requirements will increase the number of FHIR based content profiles reflecting internationally agreed standards for dedicated information entities like the International Patient Summary or an Immunization Record. As such the identified patterns are rated sufficient to cover the approaches taken by IHE and to monitor the further development.

\section{References}

[1] Tahar K, Müller C, et al. Integrating Heterogeneous Data Sources for Cross-Institutional Data Sharing: Requirements Elicitation and Management in SMITH, In: Ohno-Machado L, Séroussi B, editors. Proceedings of the MedInfo 2019. 2019 August 25-29, Lyon, France. Amsterdam: IOS Presss; Stud Health Technol Inform; 2019 Aug 21. 264:1785-1786. doi: 10.3233/SHTI190647.

[2] CEN/TC251 - Health Informatics - International patient summary EN 17269:2019.

[3] Kyburz P, Gfeller S, Bürkle T, Denecke K. Exchanging Appointment Data Among Healthcare Institutions, in: D. Hayn et al., editors, dHealth 2019 - Proceedings of the 13th Health Informatics Meets Digital health Conference; 2019 May 28-29; Vienna, Austria. Amsterdam: IOS Press; Stud Health Technol Inform; 2019. p. 260:33-40. doi:10.3233/978-1-61499-971-3-33.

[4] IHE Category: FHIR, Available at: https://wiki.ihe.net/index.php/Category:FHIR, Accessed January 31, 2021.

[5] IHE Profiles, Available at https://wiki.ihe.net/index.php/Profiles, Accessed January 31, 2021.

[6] IHE Connectathon Result Browser, Available at: https://connectathon-results.ihe.net/index.php? highlight=10, Accessed January 31, 2021.

[7] IHE profiles and supplements, Available at: https://www.ihe.net/resources/profiles/ using the respective domains, Accessed January 31, 2021.

[8] HL7 FHIR operations, Available at: https://www.hl7.org/fhir/operations.html, Accessed January 21, 2021.

[9] IHE Wiki, https://wiki.ihe.net/index.php/Mobile_Health_Document_Sharing (MHDS). 